NBER TECHNICAL PAPER SERIES

\title{
ON INFLATION AND OUTPUT \\ WITH COSTLY PRICE CHANGES: \\ A SIMPLE UNIFYING RESULT
}

Roland Bénabou

Jerzy Konieczny

Technical Paper No. 135

\section{NATIONAL BUREAU OF ECONOMIC RESEARCH 1050 Massachusetts Avenue \\ Cambridge, MA 02138 \\ May 1993}

This paper is part of NBER's research program in Economic Fluctuations. Any opinions expressed are those of the authors and not those of the National Bureau of Economic Research. 
NBER Technical Paper \#135

May 1993

\title{
ON INFLATION AND OUTPUT \\ WITH COSTLY PRICE CHANGES: \\ A SIMPLE UNIFYING RESULT
}

\begin{abstract}
We analyze the effect of inflation on the average output of monopolistic firms facing a small fixed cost of changing nominal prices. Using Taylor expansions, we derive a general closed-form solution for the slope of the long-run Phillips curve. This very simple, unifying formula allows us to evaluate and clarify the role of three key factors: the asymmetry of the profit function, the convexity of the demand function, and the discount rate. These partial equilibrium effects remain important components of any general equilibrium model with $(s, S)$ pricing.
\end{abstract}

Roland Bénabou

Department of Economics

MIT

Cambridge, MA 02138

and NBER

\author{
Jerzy Konieczny \\ Department of Economics \\ Wilfried Laurier University \\ Ontario, Canada N2L 3C5
}




\section{On Inflation and Output with Costly Price Changes: A Simple Unifying Result by Roland Bénabou and Jerzy D. Konieczny *}

We study the effect of inflation on the average output of monopolistic firms facing fixed costs of changing their nominal price. The slope of this long run Phillips curve has been analyzed by several authors, usually with specific functional forms. As a result, the literature offers a somewhat confusing array of special results. In this paper we derive a simple, explicit formula which incorporates all the effects previously identified, and determines their net impact.

Julio J. Rotemberg (1983) claims, for constant elasticity demand functions and quadratic costs, that inflation has no effect on average (log) output; we show that it actually has a negative impact. Timur Kuran (1986) finds effects of opposite signs for nonincreasing elasticity and concave increasing elasticity demand functions. Howard F. Naish (1987) uses linear and isoelastic specifications of demand and costs to demonstrate the important role played by asymmetries in the profit function around its peak. Jerzy D. Konieczny (1990) shows, more generally, that the effect of inflation on output depends not only on the skewness of profits, but also on the curvature of the demand function. But he does not provide a way of determining which factor dominates, and incorrectly claims that, at small inflation rates, the profit effect is negligible. Finally, Leif Danziger (1988) stresses the role played by discounting at small inflation rates.

The route taken in this paper is to focus on the case where the costs of price adjustment are small, which is empirically the most relevant. This allows the use of Taylor expansions 
to obtain a closed-form solution, applicable to any specification (Proposition 2). Within this unifying framework, the three effects which determine the slope of the long run Phillips curve become perfectly clear.

\section{The Model}

We consider a monopolistic firm which produces a single, perishable good and expects the inflation rate to remain constant over time. The firm can change its price at any time but doing so entails a real cost, $c>0$; the new price must be decided upon, the information disseminated, etc. $c$ also proxies for the adverse reaction of customers and competitors, not captured by the model. It is convenient to express profit and demand functions in terms of the log of the real price. The firm's problem is to choose the sequence of adjustment times $\left\{t_{\tau}\right\}_{\tau=0}^{\infty}$ and $(\log )$ real prices set at those times $\left\{P_{\tau}\right\}_{\tau=0}^{\infty}$ so as to maximize the present value of profits:

$$
V=\sum_{\tau=0}^{\infty}\left[\int_{t_{\tau}}^{t_{\tau+1}} F\left(P_{\tau}-g\left(t-t_{\tau}\right)\right) e^{-r t} d t-c e^{-r t_{\tau}}\right]
$$

where $F$ is the profit function, assumed to be strictly quasiconcave and $C_{3} ; g>0$ is the inflation rate, and $r$ is the discount rate. We shall denote by $z^{m}$ the (log) real monopoly price, and assume: $F\left(z^{m}\right)>0=F^{\prime}\left(z^{m}\right)$. The optimal pricing policy is of the $(s, S)$ type (Eytan Sheshinski and Yoram Weiss, 1977); at each adjustment date, the firm sets the nominal price so that the ( $\log$ of) real price is $S>z^{m}$; a new change occurs when inflation has eroded the real price to $s<z^{m}$. Moreover, the optimal $(s, S)$ policy satisfies the first 
order conditions: ${ }^{1}$

$$
F(S)-F(s)=r c
$$

$$
F(s)=r V
$$

Strict quasiconcavity implies that the optimum is unique and satisfies:

$$
F^{\prime}(s)>0>F^{\prime}(S) ; \quad \frac{d S}{d g}>0>\frac{d s}{d g}
$$

We now consider an economy consisting of many such firms which stagger their price changes uniformly over time. Aggregate output is then equal to the average output of a firm over its price cycle:

$$
\bar{Y}=\frac{g}{S-s} \int_{0}^{(S-s) / g} y(S-g t) d t=\frac{1}{S-s} \int_{s}^{S} y(z) d z
$$

where $y(\cdot)$ is the demand function, $y^{\prime}(\cdot)<0$. Differentiating (4) and rearranging, we obtain:

$$
\text { (5) } \frac{d \bar{Y}}{d g}=\frac{2}{F^{\prime}(s)(S-s)}\left(\frac{d S}{d g}\right)\left\{\left[\frac{y(s)+y(S)}{2}-\bar{Y}\right] F^{\prime}(s)-\left[\frac{F^{\prime}(s)+F^{\prime}(S)}{2}(y(s)-\bar{Y})\right]\right\}
$$

As both $F^{\prime}(s)$ and $y(s)-\bar{Y}$ are positive, (5) makes clear that the effect of inflation on output depends on:

(i) the skewness of the profit function (represented by the asymmetry in marginal profits, $\left.F^{\prime}(s)+F^{\prime}(S)\right)$, which determines the effect of inflation on the price bounds $s$ and $S$, 
through (2). This will be called the profit effect;

(ii) the curvature of the demand function (represented by the difference $\left(\frac{y(s)+y(S)}{2}-\bar{Y}\right)$, which determines the output effects of those changes. This will be called the demand effect.

Following Konieczny (1990), we call $F(\cdot)$ strongly left skewed if $F^{\prime}\left(z_{1}\right)<-F^{\prime}\left(z_{2}\right)$ for every $z_{1}<z^{m}<z_{2}$ such that $F\left(z_{1}\right)=F\left(z_{2}\right)$, and strongly right skewed if the opposite inequality holds. Strong skewness has a simple interpretation in the case of no discounting, where (2a) requires that $F(S)=F(s)$. If $F(\cdot)$ is strongly left skewed then, as inflation rises, $S-z^{m}$ increases slower than $z^{m}-s$. As a result, each price cycle is skewed towards lower values, so that the profit effect tends to increase output (see (5) and also (11) below). The opposite holds for $F(\cdot)$ strongly right skewed.

\section{Small Adjustment Costs or Low Inflation: A Simple Formula}

While (5) reveals the qualitative effects at work, its implicit nature makes it rather opaque and unwieldy. For instance, the effect of discounting is buried deep inside the formula. We therefore concentrate from now on on the case where the inflation rate $g$, or especially the adjustment cost $c$, is small. These cases are the most empirically relevant, and also allow us to derive closed-form solutions for $s, S$ and $d \bar{Y} / d g$. To simplify the exposition, we first consider the case where $r=0$; discounting will be incorporated in Section IV. The first-order conditions become:

$$
F(s)=F(S)
$$




$$
F(S)=\bar{F}
$$

where $\bar{F}$ denotes average profits per unit of time, net of adjustment costs: $\bar{F}=\left[\int_{a}^{S} F(z) d z-g c\right] /(S-$ $s)$. By (2') both price bounds, $s$ and $S$, approach the monopoly price $z^{m}$, as $c$ or $g$ approach zero. Define therefore:

$$
\delta \equiv S-z^{m} \ll 1 \quad \text { and } \quad \tau \equiv z^{m}-s \approx \alpha_{1} \delta+\alpha_{2} \delta^{2}
$$

Throughout the paper, $X \approx \sum_{i=0}^{n} \alpha_{i} x^{i}, \alpha_{n} \neq 0$, represents a Taylor expansion of order $n$ in $x$, i.e. stands for $X-\sum_{i=0}^{n} \alpha_{i} x^{i} \ll x^{n}$. Thus $\alpha_{1}+\alpha_{2} \delta$ represents the relative rate at which $\tau=z^{m}-s$ goes to zero in comparison to $\delta=S-z^{m}$, when $c$ or $g$ become very small. Now, for all $z$ in $[s, S]$ :

$$
F(z) \approx F\left(z^{m}\right)+\frac{F^{\prime \prime}\left(z^{m}\right)}{2}\left(z-z^{m}\right)^{2}+\frac{F^{\prime \prime \prime}\left(z^{m}\right)}{6}\left(z-z^{m}\right)^{3}
$$

Expanding (2a') to the third order in $\delta$ and substituting in $\tau \approx \delta\left(\alpha_{1}+\alpha_{2} \delta^{2}\right)$, we get:

$$
\frac{F^{\prime \prime}\left(z^{m}\right)}{2} \delta^{2}+\frac{F^{\prime \prime \prime}\left(z^{m}\right)}{6} \delta^{3} \approx \frac{F^{\prime \prime}\left(z^{m}\right)}{2} \alpha_{1}^{2} \delta^{2}+\left[F^{\prime \prime}\left(z^{m}\right) \alpha_{1} \alpha_{2}-\frac{F^{\prime \prime \prime}\left(z^{m}\right)}{6} \alpha_{1}^{3}\right] \delta^{3} .
$$

Identifying the two polynomials leads to $\alpha_{1}=1$ and $\alpha_{2}=-2 a / 3$, where $a \equiv-F^{\prime \prime \prime}\left(z^{m}\right) /\left(2 F^{\prime \prime}\left(z^{m}\right)\right)$ measures the skewness of the profit function around $z^{m}$, relative to its curvature. ${ }^{2}$ Hence:

$$
z^{m}-s=\tau \approx \delta(1-2 a \delta / 3)
$$


Next, expanding (ib'), we get:

$$
\left[F\left(z^{m}\right)+\frac{F^{\prime \prime}\left(z^{m}\right)}{2} \delta^{2}\right](\delta+\tau) \approx \int_{s}^{S}\left[F\left(z^{m}\right)+\frac{F^{\prime \prime}\left(z^{m}\right)}{2}\left(z-z^{m}\right)^{2}\right] d z-c g=F\left(z^{m}\right)(\delta+\tau)+\frac{F^{\prime \prime}\left(z^{m}\right)}{6}\left(\delta^{3}+\tau^{3}\right.
$$

Using (7), this gives for the upper band width the familiar "cubic root" expression:

$$
S-z^{m}=\delta \approx\left[-\frac{3}{2} \frac{c g}{F^{\prime \prime}\left(z^{m}\right)}\right]^{1 / 3}
$$

as in Michael Muss (1981) and Rotemberg (1983). The interpretation of the effect of each parameter on the optimal $S-z^{m}$ is straightforward. Finally, we return to output.

Expanding $y(z)$, integrating (4) and using (7) we get:

$$
\bar{Y} \approx y\left(z^{m}\right)+y^{\prime}\left(z^{m}\right) \frac{a \delta^{2}}{3}+y^{\prime \prime}\left(z^{m}\right) \frac{\delta^{2}}{6}
$$

and so

$$
\bar{Y} \approx y\left(z^{m}\right)-y^{\prime}\left(z^{m}\right) \frac{\delta^{2}}{6}\left[\frac{F^{\prime \prime \prime}\left(z^{m}\right)}{F^{\prime \prime}\left(z^{m}\right)}-\frac{y^{\prime \prime}\left(z^{m}\right)}{y^{\prime}\left(z^{m}\right)}\right]
$$

Proposition 1 Let $r=0$. Then, for small adjustment costs and/or inflation rates (gcc « $\left.-F^{\prime \prime}\left(z^{m}\right)\right)$, the slope of the long run Phillips curve is:

$$
\frac{d \bar{Y}}{d g} \approx A g^{-1 / 3}\left[\frac{F^{\prime \prime \prime}\left(z^{m}\right)}{F^{\prime \prime}\left(z^{m}\right)}-\frac{y^{\prime \prime}\left(z^{m}\right)}{y^{\prime}\left(z^{m}\right)}\right]
$$

where $A=-\frac{y^{\prime}\left(z^{m}\right)}{9}\left[-\frac{3}{2} \frac{\epsilon}{F^{\prime \prime}\left(z^{m}\right)}\right]^{2 / 3}>0$.

6 


\section{Interpretation and Examples}

The use of Taylor approximations yields explicit measures of the profit and demand effects on average output. It is intuitive, and easily verified, that $F(\cdot)$ is strongly left skewed in the neighborhood of $z^{m}$ if $F^{\prime \prime \prime}\left(z^{m}\right)<0$, and strongly right skewed if $F^{\prime \prime \prime}\left(z^{m}\right)>0$. Thus two equivalent measures of the effect of skewness on price bounds are, from (6) through (8):

$$
\frac{s+S}{2}-z^{m} \approx \frac{a \delta^{2}}{3}, \text { or } F^{\prime}(s)+F^{\prime}(S) \approx-F^{\prime \prime}\left(z^{m}\right) \frac{4 a \delta^{2}}{3}
$$

The effect of changes in the price bounds on average output depends, in turn, on the curvature of the demand function. A measure of this effect is, from (7) through (9):

$$
\frac{y(s)+y(S)}{2}-\bar{Y} \approx y^{\prime \prime}\left(z^{m}\right) \frac{\delta^{3}}{3}
$$

By Jensen's inequality, the demand effect tends to increase output if the demand function is convex, and to decrease it if demand is concave.

Proposition 1 shows how the net effect of inflation depends on the relative strength of the profit and demand effects. Konieczny (1990) claims that, at low inflation rates, the profit effect is negligible in comparison to the demand effect, because the profit function is log-quadratic, hence symmetric, up to a third order approximation. It is clear from (11) and (12) that this is not correct: both bracketed terms in (5) are of order $\delta^{3}$. This is due to the fact that the relevant asymmetry is not that of profits, but of marginal profits.

We conclude this discussion by identifying the two effects for some of the functional 
forms most commonly used in the literature. When the demand function is linear in the real price, $y(z)=\alpha-\beta e^{z}$, and marginal cost is either constant or linear, the profit and demand effects are respectively $3 A g^{-1 / 3}$ and $-A g^{-1 / 3}$, where $A$ is given by Proposition 1 . The former, which tends to make output rise with inflation, dominates the latter, which goes the other way; thus $d \bar{Y} / d g=2 A g^{-1 / 3}>0$.

When both demand $y(z)=B e^{-\beta z}$ and costs $\Phi(y)=\phi y^{a}, \alpha>0, \beta>1$ are isoelastic, the profit effect is $A g^{-1 / 3}(1-\beta-\alpha \beta)$; the demand effect is $A g^{-1 / 3} \beta$, and so $d \bar{Y} / d g=$ $A g^{-1 / 3}(1-\alpha \beta)$. The profit effect tends to decrease output, while the demand effect operates in the opposite direction. The profit effect is stronger for $\alpha \beta>1$, and in particular for non-decreasing marginal costs $(\alpha \geq 1)$. For the demand effect to dominate, marginal cost must decrease fast enough.

Rotemberg (1983) uses isoelastic demand and quadratic costs $(\alpha=2)$. Approximating profits as quadratic in log-price, he claims that inflation has no effect on average log-output, and that the same should hold for average output. This is incorrect on two counts. First, the quadratic approximation neglects the profit effect, equal to $A g^{-1 / 3}(1-3 \beta)<0$. Second, evaluating the average log-output instead of output itself amounts to omitting the demand effect, $A g^{-1 / 3} \beta>0$. The correct analysis yields $d \bar{Y} / d g<0$.

The isoelastic case also helps to identify the contributions of the elasticity of demand and of the convexity of costs to the profit and demand effects. A lower degree of returns to scale (a larger value of $\alpha$ ) accentuates the strong right skewness of the profit function, and thus tends to make output fall with inflation. Indeed, as the real price is eroded by 
inflation output increases and, with a large $\alpha$, costs rise fast; this magnifies the profit loss for prices below $z^{m}$, relative to losses for prices exceeding $z^{m}$. The effect of an increase in the elasticity of demand is more complicated. First, the demand function becomes morc convex (relative to its slope), as $-y^{\prime \prime}(z) / y(z)=\beta$. Second, the profit function becomes more strongly skewed to the right (relative to its curvature); as above, profits fall faster for prices below $z^{m}$ than for prices above $z^{m}$. The intuition here is as follows. Marginal revenue $M R(z)=-(\beta-1) B e^{-(1-\beta) z}$ and marginal cost $M C(z)=-\alpha \beta C e^{-\alpha \beta z}$ are both increasing and concave functions of the $(\log )$ real price $z$, with $M C(z)$ steeper at $z^{m}$, by the second-order condition. A higher demand elasticity can be shown to raise the difference in curvature between the two functions, relative to the difference in their slopes. This implies that marginal profits $M R(z)-M C(z)$ are steeper to the left of $z^{m}$ than to the right.

Apart from the isoelastic case, it is generally not feasible to isolate the contributions of demand and costs to the profit effect, as $\Pi^{\prime \prime \prime}\left(z^{m}\right) / \Pi^{\prime \prime}\left(z^{m}\right)$ is a very mesy combination of derivatives of both functions. In fact, our analysis shows that profits really are the proper general concept; the effects of demand and costs specifications are interdependent, and trying to separate them only leads back to the catalogue of special results from the earlier literature.

\section{A General Formula with Discounting}

When the discount rate is positive, firms care more about profits closer to the beginning of each cycle than those in the later phase. Therefore, they set the initial real price $S$ closer to the profit maximizing level $z^{m}$ than when $r=0$. The price is then allowed to deteriorate 
more before the next adjustment. In particular, discounting creates a discontinuity in the pricing rule: as $g \rightarrow 0^{+}, \delta=S-z^{m} \rightarrow 0$ but $\tau=z^{m}-s$ remains bounded from below, since $F(S)-F(s)=r c$. This results in an upward jump in the level of average output $\bar{Y}$ at $g=0^{+} ;$see Danziger (1988). As to the slope $d \bar{Y} / d g$ of the Phillips curve at any given $g>0$, it is negatively affected by discounting. Indeed by $(2), F^{\prime}(S)(d S / d g)=F^{\prime}(s)(d s / d g)$; since discounting makes $S$ closer to $z^{m}$, for small $c$ or $g$ it reduces $\left|F^{\prime}(S)\right|$ relative to $F^{\prime}(s)$. Therefore, as the inflation rate rises, the initial price increases more than the terminal one falls. This raises the average price and lowers average output (Danziger, 1988, Konieczny, 1990). To show precisely, and explicitly, how discounting combines with the profit and demand effects, we generalize Proposition 1 to the case where $r>0$. We let $g$ take any given positive value, and consider the case where the adjustment cost $c$ is small. Then, (2b) can be rewritten as:

$$
\int_{s}^{S} F(z) e^{\left(z-z^{m}\right) r / g} d z=c g e^{\delta r / g}+F(s)\left[\frac{e^{\delta r / g}-e^{-\tau r / g}}{r / g}\right]
$$

where, as before, $\delta \equiv S-z^{m} \ll 1$ and $\tau \equiv z^{m}-s \approx \alpha_{1} \delta+\alpha_{2} \delta^{2}$. Expanding again the first-order conditions (2a) and (13) to the third order in $\delta$, we obtain (see the appendix): $\alpha_{1}=1$, and $\alpha_{2}=(2 / 3)(r / g-a)$. Hence:

$$
\tau \approx \delta+\frac{2}{3}\left(\frac{r}{g}-a\right) \delta^{2}, \quad \text { or } \quad z^{m}-\frac{s+S}{2} \approx \frac{\delta^{2}}{3}\left(\frac{r}{g}-a\right)
$$


where $\delta$ is still given by (8). Expanding average output as before leads to:

$$
\bar{Y} \approx y\left(z^{m n}\right)-y^{\prime}\left(z^{m}\right) \frac{\delta^{2}}{6}\left(\frac{2 r}{g}+\frac{F^{\prime \prime \prime}\left(z^{m}\right)}{F^{\prime \prime}\left(z^{m}\right)}-\frac{y^{\prime \prime}\left(z^{m}\right)}{y^{\prime}\left(z^{m}\right)}\right)
$$

Equations (14)-(15) clearly show that positive discounting lowers the average price and raises average output, as argued above. As to its effect on the slope of the output-inflation tradeoff, it is given by:

Proposition 2 When the cost of price adjustment is small $\left(c \ll \min \left\{-F^{\prime \prime}\left(z^{m}\right) / g,-F^{\prime \prime}\left(z^{m}\right) / r\right\}\right)$, the slope of the long run Phillips curve is:

$$
\frac{d \bar{Y}}{d g} \approx A g^{-1 / 3}\left(\frac{F^{\prime \prime \prime}\left(z^{m}\right)}{F^{\prime \prime}\left(z^{m}\right)}-\frac{y^{\prime \prime}\left(z^{m}\right)}{y^{\prime}\left(z^{m}\right)}-\frac{r}{g}\right)
$$

where $A$ is the same as in Proposition 1.

This result embodies all three factors which affect the slope of the long run inflationoutput tradeoff, solely in terms of the underlying parameters of the model. It incorporates Proposition 1 as a special case and also shows that, for small enough inflation rates and $r>0$, output falls with inflation, regardless of the strength of the demand and profit effects. 


\section{From Partial to General Equilibrium}

Throughout the paper we have focused on the case of many firms operating independently of one another. General equilibrium considerations (Bénabou, 1988; 1992, Peter Diamond, 1993 and Konieczny, 1991), introduce new factors affecting the Phillips curve: firms' demand and cost functions become endogenous, and are therefore not independent of the inflation rate any morc. As the Taylor approximations approach is much harder in such models, we shall discuss their main features qualitatively. The main point is that the effects considered in this paper remain present, and form an important baseline on top of which come the general equilibrium channels.

In fact, the first implication of any general equilibrium setting is to reinforce the partial equilibrium effects on which this paper has focused, because firms' prices are strategic complements. Depending on the skewness of the profit function, the average price tends to move up or down with higher inflation. If it increases, any firm can then shift its price range up a little, without losing too many customers. This, in turn, encourages other firms to move still a little higher, and so on. The same holds for downward movements.

Consumer search is one potentially important channel through which inflation affects the equilibrium demand curve faced by firms (Bénabou $(1988,1992)$ ). By increasing price dispersion, inflation raises the return to search; demand becomes more elastic, so monopoly power is reduced, driving all real prices down, and output up. Intuition suggests, and simulations confirm, that this effect is dominant when search is relatively cheap, while the partial equilibrium effects studied above remain preeminent when information gathering 
- is relatively costly. For instance, let consumers have isoelastic preferences (indirec futility function) $U(z)=B e^{-(\beta-1) z} /(\beta-1)$, so that their demand, conditional on accepting the real price $z$, is $y(z)=B e^{-\beta z}$. In partial equilibrium, the resulting demand and profit effects imply $d \bar{Y} / d g$ $<$ 0 , assuming non-decreasing marginal costs. In a general search equilibrium, the Phillips curve remains negatively sloped when most consumers have relatively high search costs; but it becomes flatter, and eventually upward- sloping, as search costs become low enough to allow a sufficient number of buyers to take advantage of the dispersion induced by inflation. Similarly, if consumers have quadratic preferences $U(z)=\left(\alpha-\beta e^{z}\right)^{2} /(2 \beta)$, their demand in partial equilibrium is $y(z)=\alpha-\beta e^{z}$, and the balance of demand and profit effects implies $d \bar{Y} / d g>0$ (except at very low inflation rates when $r>0$ ). This slope remains positive in general equilibrium, and in fact becomes steeper for lower values of search costs.

The exit of firms, due to lower profitability at higher inflation rates, generates two other effects on demand functions, through either the pricing or the search technology. As the remaining firms have higher market share, hence a higher level of demand, they find it worthwhile to incur the adjustment cost more frequently; this dampens all other effects of inflation on the average price and output (Bénabou, 1992). On the other hand, if the thinner market makes it more difficult for customers to search (Diamond, 1993), exit lowers the elasticity of demand for the remaining firms. This increases their market power and lowers output.

Finally, Konieczny (1991) considers general equilibrium effects which operate through 
labor costs and aggregate demand. When firms face short run decreasing returns, the increased variability of prices and output due to inflation raises average costs, and thereby lowers labor plus profit income. This in turn reduces aggregate demand and equilibrium output. This decline compounds the fall which results (given his assumptions of isoelastic demand and convex costs) from the partial equilibrium effect discussed earlier.

\section{Conclusion}

We have analyzed the effect of inflation on the average output of monopolistic firms facing a small fixed cost of changing nominal prices. Using Taylor expansions, we derived a closed-form solution which can be applied to any specification. This extremely simple formula allowed us to evaluate and clarify the relative impact of the three factors which affect the inflation-output tradeoff: the asymmetry of the profit function, the convexity of the demand function, and discounting. These effects remain important components of any general equilibrium model with $(s, S)$ pricing. 


\section{Appendix: Proof of Proposition 2}

Throughout the appendix, $y=o(g(x))$ will mean that $\lim _{x \rightarrow 0}[y / g(x)]=0$. We denote $\theta \equiv r / g$ and $\varepsilon \equiv-c g / 2 F^{\prime \prime}\left(z^{m}\right) \ll 1$. Expanding $S-z^{m}$ and $s-z^{m}$ in powers of $\varepsilon$,

$$
\begin{aligned}
& S-z^{m}=\delta=\lambda \varepsilon^{\gamma}+\mu \varepsilon^{2 \gamma}+o\left(\varepsilon^{2 \gamma}\right) \\
& z^{m}-s=\tau=\lambda^{\prime} \varepsilon^{\gamma}+\mu^{\prime} \varepsilon^{2 \gamma}+o\left(\varepsilon^{2 \gamma}\right),
\end{aligned}
$$

where $\gamma$ is the smaller of the orders of $\delta$ and $\tau$ in $\varepsilon$, and $\lambda \geq 0, \lambda^{\prime} \geq 0, \lambda+\lambda^{\prime}>0$. Now, expanding $F(\cdot)$ around its maximum (and dropping the argument $z^{m}$ from here on) yields:

$$
\begin{aligned}
& F(S)=F+F^{\prime \prime} \lambda^{2} \varepsilon^{2 \gamma} / 2+\left(F^{\prime \prime} \lambda \mu+F^{\prime \prime \prime} \lambda^{3} / 6\right) \varepsilon^{3 \gamma}+o\left(\varepsilon^{3 \gamma}\right) \\
& F(s)=F+F^{\prime \prime} \lambda^{2} \varepsilon^{2 \gamma} / 2+\left(F^{\prime \prime} \lambda^{\prime} \mu^{\prime}-F^{\prime \prime \prime} \lambda^{\prime 3} / 6\right) \varepsilon^{3 \gamma}+o\left(\varepsilon^{3 \gamma}\right)
\end{aligned}
$$

Hence, from (2a):

$(\mathbf{G g}) \cdot\left(\lambda^{2}-\lambda^{\prime 2}\right) \varepsilon^{2 \gamma} / 2+\left[F^{\prime \prime} \cdot\left(\lambda \mu-\lambda^{\prime} \mu^{\prime}\right)+F^{\prime \prime \prime} \cdot\left(\lambda^{3}+\lambda^{\prime 3}\right) / 6\right] \varepsilon^{3 \gamma}+o\left(\varepsilon^{3 \gamma}\right)=r c=-2 F^{\prime \prime} \varepsilon \theta$

Next, expand the expressions on the left and right-hand side of equation (13):

$$
\begin{aligned}
F(z) e^{\theta\left(z-z^{m}\right)} & =F\left(z^{m}\right)+\left(z-z^{m}\right) \theta F+\left(z-z^{m}\right)^{2}\left[F^{\prime \prime}+\theta^{2} F\right] / 2+o\left(\left(z-z^{m}\right)^{2}\right) \\
\int_{s}^{S} F(z) e^{\theta\left(z-z^{m}\right)} d z & =(\delta+\tau) F+\left(\delta^{2}-\tau^{2}\right) \theta F / 2+\left(\delta^{3}+\tau^{3}\right)\left(F^{\prime \prime}+\theta^{2} F\right) / 6+o\left(\varepsilon^{3 \gamma}\right) \\
\left(e^{\theta \delta}-e^{-\theta \tau}\right) / \theta & =(\delta+\tau)+\left(\delta^{2}-\tau^{2}\right) \theta / 2+\left(\delta^{3}+\tau^{3}\right) \theta^{2} / 6+o\left(\varepsilon^{3 \gamma}\right) .
\end{aligned}
$$

Substituting into (13) and neglecting terms smaller than $o\left(\varepsilon^{3 \gamma}\right)$ we get, after some rearrangement: 
$\left(20 \ell F^{\prime \prime} / 6\right)(\delta+\tau)\left(\delta^{2}-\delta \tau-2 \tau^{2}\right)+o\left(\varepsilon^{3 \gamma}\right)=-2 F^{\prime \prime} \varepsilon\left(1+\theta \delta+\theta^{2} \delta^{2} / 2+\theta^{3} \delta^{3} / 6\right)+o\left(\varepsilon^{3 \gamma}\right)$

Substituting from (A1): $(\delta+\tau)\left(\delta^{2}-\delta \tau-2 \tau^{2}\right)=\left(\lambda+\lambda^{\prime}\right)\left(\lambda^{2}-\lambda \lambda^{\prime}-2 \lambda^{\prime 2}\right) \varepsilon^{3 \gamma} / 6+o\left(\varepsilon^{3 \gamma}\right)$, and so:

$$
-2 F^{\prime \prime} \varepsilon(1+o(1))=F^{\prime \prime}\left(\lambda+\lambda^{\prime}\right)\left(\lambda^{2}-\lambda \lambda^{\prime}-2 \lambda^{2}\right) \varepsilon^{3 \gamma} / 6+o\left(\varepsilon^{3 \gamma}\right) .
$$

Assume first that $\lambda \neq \lambda^{\prime}$. Then, by (A3), $\gamma=1 / 2$ and so (A4) becomes: $-2 F^{\prime \prime} \varepsilon+o(\varepsilon)=$ $\Omega \varepsilon^{3 / 2}+o\left(\varepsilon^{3 / 2}\right)$, where $\Omega=F^{\prime \prime}\left(\lambda+\lambda^{\prime}\right)\left(\lambda^{2}-\lambda \lambda^{\prime}-2 \lambda^{2}\right) / 6$. Hence: $-2+o(1)=\Omega \varepsilon^{1 / 2} / F^{\prime \prime}+$ $o\left(\varepsilon^{1 / 2}\right)$; but the right hand side tends to zero with $\varepsilon$ while the left hand side does not, a contradiction. So it must be that $\lambda=\lambda^{\prime}$ necessarily. Using this, (A3)-(A4) become:

$$
\begin{aligned}
{\left[F^{\prime \prime}\left(\mu-\mu^{\prime}\right)+F^{\prime \prime \prime} \lambda^{3} / 3\right] \varepsilon^{3 \gamma}+o\left(\varepsilon^{3 \gamma}\right) } & =-2 F^{\prime \prime} \varepsilon \theta \\
-2 F^{\prime \prime} \varepsilon+o(\varepsilon) & =F^{\prime \prime}\left(-4 \lambda^{3}\right) \varepsilon^{3 \gamma} / 6+o\left(\varepsilon^{3 \gamma}\right)
\end{aligned}
$$

Now, (A6) implies $\lambda^{3}=3$ and $\gamma=1 / 3$. Inserting in (A5), we obtain $\mu-\mu^{\prime} \approx 2 \lambda^{2}(a-\theta) / 3$. So, from (A1):

$$
\begin{aligned}
& S-z^{m}=\delta=(3 \varepsilon)^{1 / 3}+\mu \varepsilon^{2 / 3}+o\left(\varepsilon^{2 / 3}\right) \\
& z^{m}-s=\tau=(3 \varepsilon)^{1 / 3}+\mu \varepsilon^{2 / 3}+2(\theta-a)(3 \varepsilon)^{2 / 3} / 3+o(\varepsilon)^{2 / 3} .
\end{aligned}
$$

Finally, (A7)-(A8) imply (8) and (14); (15) and (16) follow immediately. Q.E.D. 


\section{References}

Bénabou, Roland, "Search, Price Setting and Inflation," Review of Economic Studies, July $1988,55: 353-373$.

Bénabou, Roland, "Inflation and Efficiency in Search Markets," Review of Economic Studies, April 1992, 59: 299-329.

Diamond, Peter, "Search, Sticky Prices and Inflation," Review of Economic Studies, January $1993,59: 53-68$.

Danziger, Leif, "Costs of Price Adjustment and the Welfare Economics of Inflation and Disinflation," American Economic Review, September 1988, 78:, 633-646.

Dixit, Avinash K., "Analytical Approximations in Models of Hysteresis," Review of Economic Studies, January 1991, 58: 141-151.

Konieczny, Jerzy D., "Inflation, Output and Labour Productivity when Prices are Changed Infrequently," Economica, May 1990, 57: 201-218.

Konieczny, Jerzy D., “The Optimal Rate of Inflation with Fixed Costs of Changing Prices," mimeo, Wilfrid Laurier University, 1991.

Kuran, Timur, "Price Adjustment Costs, Anticipated Inflation and Output," Quarterly Journal of Economics, May 1986, 101: 407-418.

Mussa, Michael, "Sticky Prices and Disequilibrium Adjustment in a Rational Model of the Inflationary Process," American Economic Review, December 1981, 71: 10201027.

Naish, Howard F., "Price Adjustment Costs and the Output- Inflation Tradeoff," 
Economica, May 1986, 53: 219-230.

Rotemberg, Julio J., "Aggregate Consequences of Fixed Costs of Price Adjustment," American Economic Review, June 1983, 73: 433-436.

Sheshinski, Eytan and Weiss, Yoram, "Inflation and the Costs of Price Adjustment," Review of Economic Studies, June 1977, 44: 287-303. 


\section{Notes}

${ }^{0}$ Bénabou, Department of Economics, Massachusetts Institute of Technology, Cambridge, MA 02139, and NBER; Konieczny, Department of Economics, Wilfried Laurier University, Waterloo, Ontario, Canada N2L 3C5. Bénabou gratefully acknowledges financial support from the National Science Foundation.

${ }^{1}$ Additional assumptions on $F(\cdot)$ and $c$ are needed so that $V \geq 0$ when the optimal policy is followed. The case of deflation is obtained by permuting $S$ and $s$ throughout the paper.

${ }^{2}$ Throughout the paper we shall assume $F^{\prime \prime}\left(z^{m}\right)<0$, but the results can be generalized to $F^{\prime \prime}\left(z^{m}\right)=0$. What matters then is the ratio $F^{(k+l)}\left(z^{m}\right) / F^{(k)}\left(z^{m}\right)$, where $F^{(k)}\left(z^{m}\right)$ is the first non-zero derivative of even order, and $F^{(k+l)}\left(z^{m}\right)$ the next non-zero derivative of odd order. A proof is available upon request. 